\title{
The Effect of Pro-Neurogenic Gene Expression on Adult Subventricular Zone Precursor Cell Recruitment and Fate Determination After Excitotoxic Brain Injury
}

\author{
Jones $\mathrm{KS}^{1}$, Connor BJ
}

Despite the presence of on-going neurogenesis in the adult mammalian brain, neurons are generally not replaced after injury. Using a rodent model of excitotoxic cell loss and retroviral (RV) lineage tracing, we previously demonstrated transient recruitment of precursor cells from the subventricular zone (SVZ) into the lesioned striatum. In the current study we determined that these cells included migratory neuroblasts and oligodendrocyte precursor cells (OPC), with the predominant response from glial cells. We attempted to override this glial response by ectopic expression of the pro-neurogenic genes Pax6 or Dlx2 in the adult rat SVZ following quinolinic acid lesioning. RV-Dlx2 over-expression stimulated repair at a previously non-neurogenic time point by enhancing neuroblast recruitment and the percentage of cells that retained a neuronal fate within the lesioned area, compared to RV-GFP controls. RV-Pax6 expression was unsuccessful at inhibiting glial fate and intriguingly, increased OPC cell numbers with no change in neuronal recruitment. These findings suggest that gene choice is important when attempting to augment endogenous repair as the lesioned environment can overcome pro-neurogenic gene expression. Dlx 2 over-expression however was able to partially overcome an anti-neuronal environment and therefore is a promising candidate for further study of striatal regeneration.

\section{Key Words: Adult neurogenesis, Pax6, Dlx2, Regeneration, Oligodendrocyte progenitor cell, Neuroblast}

\section{Introduction}

Compensatory adult neurogenesis has been demonstrated in many models of brain injury and neurodegeneration, including stroke, trauma, epilepsy, excitotoxic lesioning, Huntington's and Parkinson's disease ${ }^{[1-9]}$. However, complete neuronal repair is yet to be achieved. In the normal adult mammalian brain, neurogenesis is predominantly observed from the subventricular zone (SVZ) of the lateral ventricles and throughout the olfactory bulb system, as well as the dentate gyrus of the hippocampus. Specifically in the SVZ, slow dividing Type $B$ stem cells generate rapidly proliferating Type $\mathrm{C}$ cells (transiently amplifying precursors or TAPs) that can generate both neuroblasts and oligodendrocytes $^{[7,10]}$. TAPs have been found to be heterogeneous and express combinations of the pro-neurogenic genes Mashl, Pax6, Ngn2, Dlx2 and the oligodendrocyte lineage gene Olig $2^{[2,11-16]}$. Neural cell loss or injury in the adult brain can alter endogenous neurogenesis and elicit a compensatory repair process. Specifically, alterations in the expression of Mash1, Pax6, Ngn2, Dlx2 and/or Olig2 have been observed in the adult SVZ and parenchyma in various models of neural cell loss, consistent with their potential roles in the endogenous repair process ${ }^{[2,8,9,16-19]}$.

If endogenous repair is to be restorative, attraction of the appropriate cellular phenotype to repopulate and repair damaged areas of the brain is essential. After injury, SVZ neural progenitor cells (NPCs) have been found to be redirected to areas of neural cell loss and either, 1) stay in their original neuronal lineage $\operatorname{program}^{[20,21]} ; 2$ ) change their lineage program to a different neuronal subtype ${ }^{[22,23]}$; or 3 ) have their lineage altered to a different cell type altogether ${ }^{[24]}$. However, the plasticity of these cell lineages differ between injury and disease models in the appropriate recruitment and differentiation of sub-type specific cells. Why this happens is not well understood. We previously showed that quinolinic acid (QA) -induced striatal cell loss stimulates a transient neurogenic response from SVZ-derived precursor cells, with increased proliferation and redirected migration of cells away from the SVZ and rostral migratory stream to the injury site ${ }^{[6]}$. Retrovirus (RV) GFP lineage tracing found the phenotype of redirected cells appeared to change over time from a neuronal to glial morphology ${ }^{[25]}$. Classically, endogenous repair studies have focused on the proliferation of SVZ progenitors labelled with Bromodeoxyuridine or with RV-GFP, to track the migration of $\mathrm{DCX}+$ precursor cells to areas of neural damage and determine the neural phenotypes generated, while ignoring non-DCX+ migratory cells $^{[6,22,23,25,26]}$. To address this, the current study examined the phenotypic profiles of all RV-GFP labelled cells migrating from the SVZ into the QA lesioned striatum over time. Interestingly, GFP+ cells observed in the injured striatum included migratory neuroblasts as well as bipolar Olig2+ cells, with the predominant response observed from glial cells.

We attempted to override this glial response by ectopic expression of the pro-neurogenic genes, Pax6 or Dlx2 in the adult rat SVZ following QA acid lesioning. Pro-neurogenic transcription factor delivery has been examined as a way to enhance a neurogenic response following neural injury ${ }^{[2,17-19,27,28]}$. Based on the presence 
of oligodendrocyte precursor cells (OPCs) in response to QA-induced striatal cell loss, we investigated the effect of over-expressing the proneurogenic factors Pax6 and Dlx2. Studies have demonstrated the requirement of Pax6 as a 'master regulator' of neurogenesis in the adult brain. Pax6 acts to suppress the glial transcription factor Olig2 in the adult SVZ and to reprogram postnatal glia and reactive astrocytes into neurons ${ }^{[12,13,15,29,30]}$. Repression of Olig2 or overexpression of Pax6 was able to promote neuroblast generation in a cortical stab wound injury and post striatal ischemia ${ }^{[18,19]}$. Olig2 has also been shown to interfere with $D l x 2$ expression and neuroblast fate in the normal SVZ ${ }^{[31]}$. Therefore, in order to determine if the observed gliogenic fate post QA lesioning could be overcome, we delivered retrovirus expressing either $P a x 6$ or $D l x 2$ with $G F P$ directly to SVZ precursor cells at time points where significant progenitor cell recruitment had been observed. Interestingly, only RV-Dlx2 overexpression enhanced both neuroblast recruitment and the percentage of recruited cells that retained a neuronal fate when compared to RVGFP control animals. Surprisingly, RV-Pax6 expression resulted in increased OPC numbers with no change in neurogenesis when compared to controls. These findings suggest that signals released from damaged tissue can selectively override pro-neurogenic gene expression. Therefore, a better understanding of interactions between neural precursor cells and inflammatory signals is required in order to successfully regenerate cells endogenously for injury or disease.

\section{Materials and Methods}

All experimental protocol were approved by the University of Auckland. Animal work was carried out with strict accordance to guidelines set by the University of Auckland Animal Ethics Committee in accordance with the New Zealand Animal Welfare Act 1999 and conformed to international guidelines for the ethical use of animals. Retroviral generation was approved by the University of Auckland and conformed to the Environmental Protection Authority of New Zealand.

\section{Retroviral generation}

pMXIG-GFP, pMXIG-Dlx2-GFP and pMXIG-Pax6-GFP plasmids were kindly donated by Professor Magdalena Gotz (Department of Physiological Genomics, Ludwig-Maximilians University, Munich, Germany $)^{[12,13,29]}$. RV-GFP, RV-Pax6-GFP and RV-Dlx2-GFP retroviral particles were generated and concentrated as per previously described methods ${ }^{[25]}$.

\section{Surgical procedures}

Adult male Wistar rats weighing 250-300g (three months of age; University of Auckland Vernon Jansen Unit) were used in this study. The animals were housed in a temperature and humidity controlled room that was kept on a 12 hour light and dark cycle. Food and water were available ad libitum. Animal welfare was monitored daily. Every effort was made to minimize the number of animals used and their suffering. For excitotoxic lesions, 400nl of fresh 50nM quinolinic acid (QA) $\mathrm{pH} 7.4$ was injected unilaterally into the striatum at the following coordinates: $\mathrm{AP}+0.7 \mathrm{~mm}$, ML $-2.5 \mathrm{~mm}$ relative to bregma, and DV $-5.0 \mathrm{~mm}$ relative to dura. Control animals did not receive a sham injection as our previous findings have demonstrated that sham surgery resulted in no significant difference to SVZ proliferation or neural precursor cell migration ${ }^{[25]}$. For $\mathrm{RV}$ injections, $2 \mu \mathrm{l}$ of concentrated retrovirus (RV-GFP, RV-Pax6-GFP or RV-Dlx2-GFP, titre $1 \times 10^{8}$ colony forming units $(\mathrm{cfu}) / \mathrm{ml}$ ) containing $80 \mu \mathrm{g} / \mathrm{ml}$ polybrene) was injected unilaterally at the following co-ordinates: AP $+0.2 \mathrm{~mm}$, ML $-1.7 \mathrm{~mm}$, relative to bregma and DV $-3.4 \mathrm{~mm}$ relative to dura. RV-GFP was injected two days prior to lesioning, on the day of lesioning and two, three, five and seven days post lesion $(n=3-5$ per time point $)^{[25]}$. RV-Pax6-GFP and RV- Dlx2-GFP were injected on the day of lesioning or two days post lesion $(n=5-6$ per virus per time point). RV control animals received an injection of either RV-Pax6GFP or RV-Dlx2-GFP into the SVZ but received no QA or sham lesion ( $\mathrm{n}=3-4)$. All animals, including controls, were transcardially perfused five days post RV injection to allow the same amount of time for cells to respond to lesion-induced signals and migrate. Perfusion was with ice cold $0.9 \%$ saline, followed by $400 \mathrm{ml} 4 \%$ paraformaldehyde in $0.1 \mathrm{M}$ phosphate buffer $\mathrm{pH}$ 7.4. Brains were removed and post fixed overnight at $4^{\circ} \mathrm{C}$ in $4 \%$ paraformaldehyde, then cryoprotected in $30 \%$ sucrose in $0.1 \mathrm{M}$ phosphate buffer $\mathrm{pH}$ 7.2. Sectioning was carried out in sagittal orientation with $40 \mu \mathrm{m}$ between each section.

\section{Immunohistochemistry}

Sections were washed $3 \times 5$ minutes in $1 \mathrm{X}$ PBS and $3 \times 5$ minutes in $1 \mathrm{X}$ PBS $+0.2 \%$ Triton $\mathrm{X}-100$. Primary antibodies were incubated at room temperature overnight in immunobuffer containing $3 \%$ donkey or goat serum in $1 \mathrm{X}$ PBS $+0.2 \%$ Triton. Primary antibodies used were: GFP (chicken polyclonal, 1:500, Abcam), GFP (rabbit polyclonal, 1:1000, Abcam), Doublecortin C-terminus (goat polyclonal, 1:300, Santa Cruz), NG2 (rabbit polyclonal, 1:300, Chemicon), GFAP (mouse monoclonal, 1:2000, Sigma), Pax6 (rabbit polyclonal, 1:1000, Covance), Dlx2 (rabbit polyclonal, 1:500, Abcam), Olig2 (rabbit polyclonal, 1:500, Chemicon), Brg1 (mouse monoclonal, Santa Cruz 1:100). Sections were then incubated at room temperature for 4 hours in secondary antibodies in immunobuffer. Secondary conjugated antibodies used were goat antichicken Alexa Fluor 488, donkey anti-rabbit Alexa Fluor 488, donkey anti-goat Alexa Fluor 594, donkey anti-rabbit Alexa Fluor 594, donkey anti-mouse Alexa Fluor 647 and donkey anti-rabbit Alexa Fluor 647 (all 1:500, Invitrogen).

\section{Quantitative PCR}

SVZ tissue was isolated from the brains of QA lesioned animals at 1, 2, 3 and 7 days post lesion $(n=3-4)$. RNA was extracted using the Qiagen Lipid Tissue RNeasy Mini Kit and cDNA synthesized using the SA Bioscience RT ${ }^{2}$ First Strand Kit with 400ng purified RNA. Quantitative SYBRgreen PCR was performed on an ABI 7900HT machine and the $\mathrm{RT}^{2}$ Profiler PCR Array System using a customised SA Biosciences Rat Neurogenesis and Neural Stem Cell PCR Array (PARN 404). SA Bioscience software calculated fold regulatory changes using the $\Delta \Delta \mathrm{Ct}$ method $^{[32]}$ between gene expression in the QA lesioned SVZ compared to control SVZ, normalised to the arthritic mean $\mathrm{Ct}$ of the three most stable housekeeping genes of five run (Rplp1 - Ribosomal protein, large P1, Rpll3a - Ribosomal protein L13A and Ldha - Lactate dehydrogenase A. Also run were Hprt1 - Hypoxanthine phosphoribosyltransferase 1 and Actb - Beta actin).

\section{Microscopy}

GFP-labelled cell counts and morphological analysis was performed under a 40x oil lens on a Zeiss Axioplan fluorescent microscope. DCX, NG2, GFAP, Dlx2, Pax6 and Olig2 immunostaining was imaged at $40 \mathrm{x}$ on a Zeiss inverted LSM fluorescent confocal microscope in $\mathrm{z}$ series with $3.4 \mu \mathrm{m}$ between each slice, with a tile scan to cover the entire striatum. 


\section{Statistical Analysis}

The number of GFP-labelled cells observed in the lesioned striatum was totalled from 12 sections per animal, per time point, per virus. Cell numbers were totalled per animal and an average GFP-labelled cells per section calculated. For RV-GFP morphology analysis over time a two way ANOVA was performed. For comparisons of RVGFP-labelled cells per timepoint a one way ANOVA followed by an all-pair wise Dunnet's multiple comparison post hoc test was performed to compare between groups. GFP-labelled cell coexpression with DCX, Olig2, NG2 or GFAP was quantified from three sections per animal, per time point, per virus. Group values were averaged and reported as the mean $+/$ - standard error of the mean. For comparisons between RV-GFP, RV-Dlx2-GFP and RV-Pax6GFP labelled cells, a one way ANOVA followed by an all-pair wise Dunnet's multiple comparison post hoc test to compare between groups was used. Control GFP (no QA lesion), day 0 and day 2 RV injection groups were analyzed separately. For qPCR, the normalized fold changes for each group of animals was compared to the normalized fold change of the control group using the student's t test, provided by the SA Biosciences software. Results were considered significant if $\mathrm{p}<0.05$.

\section{Results}

Excitotoxin-induced striatal cell loss stimulates recruitment of neuroblasts and oligodendrocyte precursor cells from the subventricular zone into the lesioned striatum

Replication incompetent, pseudotyped GFP retrovirus was injected into the SVZ of QA lesioned animals or non-lesioned controls at a
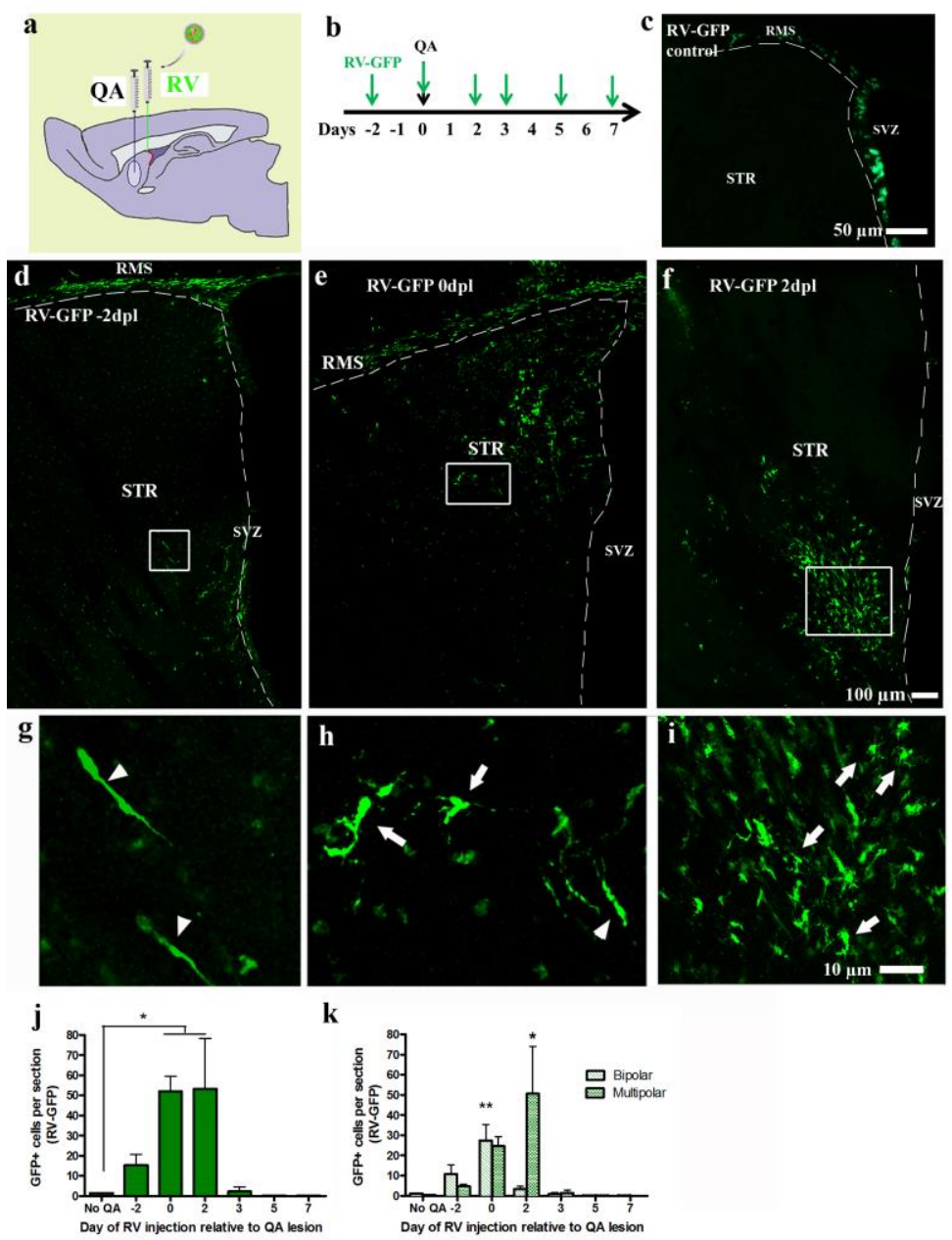

k

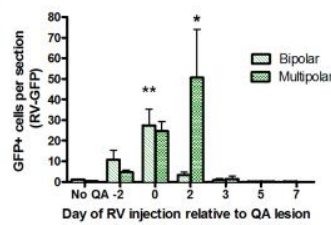

range of time points prior to or following QA injection (Figure 1a-b). Retrovirus has previously been shown to only infect fast dividing cells, including in vivo in the adult rodent $S V Z^{[33-36]}$, allowing birth dating and tracking of migration of newborn SVZ-derived precursor cells. Sham-injected $(0.9 \%$ saline $)$ controls were carried out previously ${ }^{[25]}$ and no striatal migration from SVZ-labelled progenitors was found, with GFP labelled cells remaining within the RMS and migrating normally to the olfactory bulb. Therefore, no chemotaxic property due to injury or inflammation was caused by the surgery alone. To minimize the number of animals used in this study sham controls were not repeated. GFP+ cells recruited into the QA lesioned striatum were quantified 5 days post RV-GFP injection to allow time for migration. In unlesioned control animals GFP cells were found in the SVZ, throughout the RMS (Figure 1c) and olfactory bulb but not in the striatum. In lesioned animals, in agreement with our previous observations ${ }^{[25]}$, GFP-labelled cell recruitment was observed by cells born either two days prior to QA lesioning through to 7 days following QA lesioning, with both bipolar and multipolar phenotypes observed (Figure 1d-i). Significantly more GFP-labelled cells were recruited into the QA lesioned striatum from SVZ progenitors that were born on the day of QA lesioning or two days post QA lesion when compared to controls (Figure 1j; one way ANOVA=0.0032; Dunnett's post hoc test between time points and control $\mathrm{p}<0.05$ ). At 3,5 and $7 \mathrm{dpl}$ (days post lesion) small numbers of GFP+ cells labelled were found in the striatum, SVZ and RMS, but the number of GFP cells in the striatum was not significantly different compared to non-lesioned controls.

GFP-labelled cells exhibited either bipolar or multipolar phenotypes $^{[37]}$ and the ratio of these cell types was altered in a temporal manner ${ }^{[25]}$ (Figure 1d-i, k), with a significant change in morphology observed over time (two way ANOVA F6,51=2.941 $\mathrm{p}=0.019)$. GFP+ cells labelled on the day of QA lesioning exhibited similar numbers of bipolar and multipolar morphologies, with significantly more bipolar cells in the striatum compared to controls (Figure 1k; one way ANOVA $\mathrm{p}=0.0016$, Dunnett's post hoc test $\mathrm{p}<0.01)$. In contrast, there were significantly more multipolar cells labelled 2 days following QA lesioning when compared to controls (Figure 1k; one way ANOVA p=0.013; Dunnett's post hoc test $\mathrm{p}<0.05)$. These results suggest that a switch in cell fate may be occurring within the QA lesioned striatum over time.

\footnotetext{
Figure 1: GFP-labelled cells from the SVZ are recruited into the QA-lesioned striatum and are both bipolar and multipolar. $(a-b)$ Diagram and timeline of $Q A$ and RV-GFP injections. GFP-positive cells (green) are found in the SVZ and $R M S$ of non-lesioned controls (c) and in the SVZ, RMS and striatum of lesioned animals (d-f). Confocal images showing GFP cells recruited into the striatum (STR, outlined) labelled in the SVZ two days prior (d), on the day (e), or two days post $Q A$ lesioning $(f)$. ( $g$-i) Magnified images of boxes from $d-f$ showing cell morphology. ( $j-k$ ) Graphs demonstrating ( $j$ ) the number of GFP-labelled cells per striatal section (12 sections per animal, 3-5 animals per group, one way ANOVA. * $P<0.05)$ and $(k)$ the subdivision of total GFP-labelled cells per section into bipolar and multipolar phenotypes (one way ANOVA, * $p<0.05$, ** $p<0.01$ ) over time (two way ANOVA $p<0.05$ ). Scale bar $c=50 \mu \mathrm{m}, d-f=100$ $\mu \mathrm{m}$ and $\mathrm{g}-\mathrm{I}=10 \mu \mathrm{m}$.
} 
Bipolar cells recruited from the SVZ are routinely described as neuroblasts. Interestingly, while we observed a significant increase in the number of DCX/GFP labelled cells migrating into the striatum from cells labelled on the day of QA lesioning compared to unlesioned controls (Figure 2a, c and g; one way ANOVA $\mathrm{p}<0.0001$; Dunnett's post hoc test $\mathrm{p}<0.001)$, characterization also indicated the recruitment of bipolar OLIG2/GFP labelled OPCs from the SVZ (Figure $2 b$ and d). GFP-labelled multipolar cells were found to almost exclusively co-label with the oligodendrocyte markers, OLIG2 and/or NG2 (Figure 2b, e and f). However, both bipolar and multipolar GFPlabelled cell populations contained lineage negative cells that could not be characterized with any of the markers examined including DCX, NG2, GFAP, OLIG2, MASH1, DLX2, or PAX6 (Figure 2h-i). Striatal lineage negative cells were generated from GFP-positive cells at all time points following QA lesioning and were especially prominent in the bipolar cell population. While positive staining was observed with MASH1/GFP, DLX2/GFP and OLIG2/GFP in cells within the SVZ (Supplementary Figure 1) and for DLX2/GFP and DCX/GFP throughout the RMS (Supplementary Figure 2), RV-GFPlabelled cells in the striatum were never found to express either MASH1 or PAX6 indicating either specific recruitment of subtypes of neural precursor cells in the different regions of the brain or downregulation of specific pro-neurogenic genes in recruited cells. Further, GFAP expression was never observed in any GFP-labelled cells in either the striatum or the SVZ indicating that RV-GFP was not transducing slow dividing Type B stem cells in the SVZ or proliferating GFAP+ glia and that there was no recruitment of GFAP+ glia or stem cells from the SVZ into the lesioned striatum (Supplementary Figure 7). DCX has previously been observed to colabel with NG2 in the cortex, however confocal imaging confirmed that DCX+ neuroblasts in the SVZ and migrating DCX+ cells in the lesioned striatum did not label with NG2 (Supplementary Figure 3), confirming their neuronal fate.
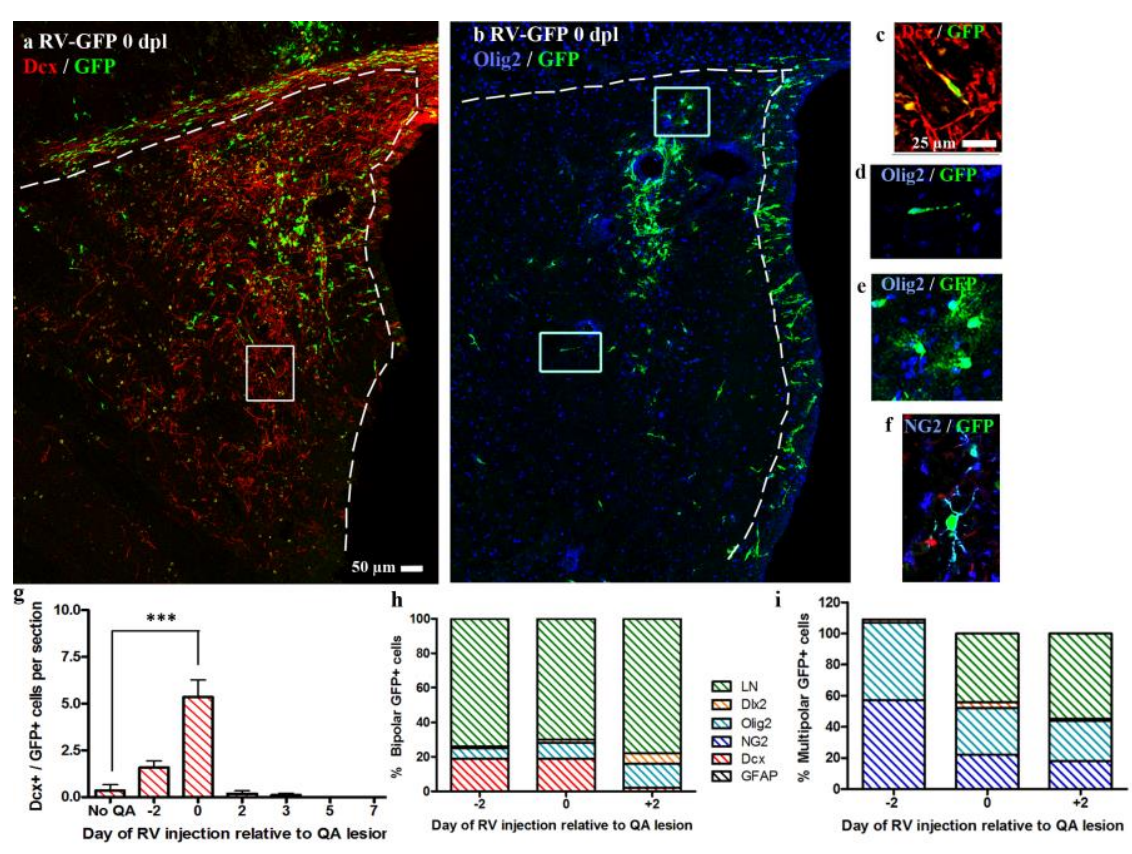

Figure 2: GFP-labelled cells recruited into the QA-lesioned striatum are neuroblasts and oligodendrocyte precursor cells. Striatal GFP cells (green, outlined area) express (a) DCX (red) and (b) OLIG2 (blue). Bipolar GFP cells can be either neuronal or oligodendrocyle lineage. (c) A bipolar DCX/GFP neuroblast (from box in a), (d) a bipolar OLIG2/GFP oligodendrocyte precursor cell (from the lower boxed region in b). Many multipolar GFP cells are oligodendrocyte precursor cells. (e) OLIG2+ multipolar GFP cells (from the upper boxed region in b) and (f) NG2+ (blue) / GFP+ (green) cells. (g) Graph demonstrating the number of DCX/GFP cells per striatal section (one way ANOVA $p<0.0001$ ). ( $h$ and i) Graphs demonstrating the percentage of bipolar $(h)$ and multipolar (i) GFP-labelled cells expressing DLX2, OLIG2, NG2, DCX, GFAP or lineage negative ( $L N)$. Scale bars $a-b=50 \mu \mathrm{m}, c-f=25 \mu \mathrm{m}$.

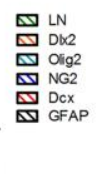

$R V$-Pax6 over-expression alters the number of striatal GFP cells

Previous studies have shown Pax6 and Dlx2 to have a potent neurogenic effect in the adult brain, and Pax6 coupled with Olig2 repression able to promote neurogenesis in injury models $^{[13,18,19,29-31]}$. Based on these previous studies and our lineage tracing results, we investigated whether RV delivery of the proneurogenic genes Pax6 or Dlx2 directly to precursor cells in the SVZ could overcome the predominantly gliogenic fate of recruited cells Pax6 or Dlx2 over-expression would reprogram lineage negative cells towards a neuronal fate. RV-Dlx2-GFP or RV-Pax6-GFP was injected into the SVZ of non-lesioned control animals and reliable co-expression of transgenes with GFP was observed (Supplementary Fure 4a-c and 5a-c). GFP+ cells remained in the SVZ and RMS of unlesioned animals and did not migrate into the striatum Supplementary Figure $6^{[25]}$ ). RV-Dlx2-GFP or RV-Pax6-GFP was inected into the SVZ either on the day of QA lesioning or 2 days (Figure 3a), the time points when significant recruitment of GFP-labelled cells was previously observed (Figure j). To determine the effect of Pax6 or Dlx2 on the recruitment of antified in the striatum for both RV-Pax6 and RV-Dlx2 treated DCX, OLIG2, NG2 and GFAP were also the effect of Pax6 or Dlx2 over-expression on precursor cell lineage following recruitment to the striatum.

Surprisingly, over-expression of Dlx2 and Pax6 gave very different results in relation to the number of GFP-labelled cells recruited to the QA lesioned striatum, even though the viruses had identical titres and therefore infected a similar number of cells upon injection into the SVZ. Percentages of cells exhibiting a neuronal fate once recruited into the damaged area were also different between groups. We observed that injection of RV-Pax6 in the SVZ on the day of QA lesioning (day 0) led to a significant reduction in the total number of GFP labelled cells recruited to the lesioned striatum (Figure 3b, red bars), including a decrease in the number of bipolar cells (Figure 3c) and DCX+ neuroblasts (Figure 3d) when compared to RVGFP controls (green bars). However, when RVPax6 was injected two days post lesion, an increase in the recruitment of GFP-labelled cells to the striatum was observed (Figure $3 b$ ).

Interestingly, the morphology of these cells was predominantly not bipolar (Figure 3c) and the number of DCX+ cells was unchanged when compared to RV-GFP control animals (Figure 3d). Instead, we observed a significant increase in a multipolar phenotype of GFP-labelled cells in the lesioned striatum in animals injected with RV-Pax6 two days post lesion (Figure 3f) and an increase in NG2 cell number when compared to RV-GFP controls (Figure 3g). However, when the percentage of striatal GFP cells coexpressing subtype specific markers was examined following RV-Pax6 over-expression, we observed that Pax6-GFP+ cells redirected into the QA lesioned striatum retained the same fate profile as RV-GFP controls with no 
significant alteration observed in the percentage of bipolar cells that expressed either OLIG2 or DCX (Figure 3j-k) or percentage of multipolar cells expressing NG2 when compared to RV-GFP control animals (Figure 31). These results indicate that Pax6 over-expression was unable to promote SVZ-derived precursor cells to a neuronal fate following recruitment into the QA lesioned striatum.

Dlx2 enhances neuroblast recruitment and retention of a neuronal fate in the QA-lesioned striatum

In contrast to Pax6, injection of RV-Dlx2 in the SVZ had no effect on the total number of GFP-labelled cells recruited into the striatum at either time point examined (Figure 3b, blue bars). Further, RV-Dlx2 had no effect on the morphology of GFP+ cells or on DCX+, OLIG2+ or $\mathrm{NG} 2+$ cell numbers when injected on the day of QA lesioning (Day 0, Figure 3c-g). However, we observed that injection of RV-Dlx 22 days following lesioning significantly increased both the number of bipolar GFP-labelled cells (Figure 3c) and the number of DCX/GFP neuroblasts compared to controls (Figure 3d) while not changing the number of multipolar cells or affecting OLIG2 or NG2 cell number (Figure 3e-g). Notably, when labelled at 2 days post lesion, the percentage of bipolar cells was significantly increased in the striatum (Figure $3 \mathrm{~h}$ ) and the percentage of DCX/GFP neuroblasts was also significantly increased to $\sim 25 \%$ of the bipolar GFP+ population when compared to controls (Figure $3 \mathrm{j}, \mathrm{m}-\mathrm{o}$ ). This occurred in conjunction with a significant reduction in multipolar cell fate (Figure 3i), while percentages of OLIG2/GFP and NG2/GFP expressing cells remained unchanged (Figure 3k-1). This may indicate that ectopic $D l x 2$ expression can induce lineage negative GFP cells to a neuronal fate. a

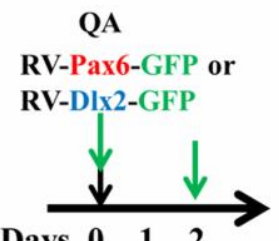

Days 0112

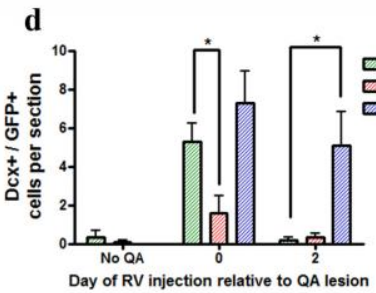

g

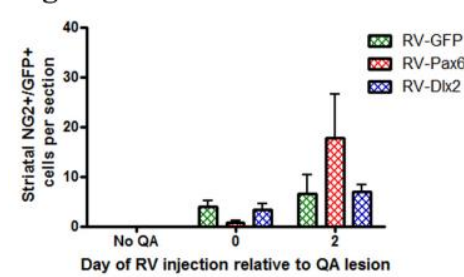

j
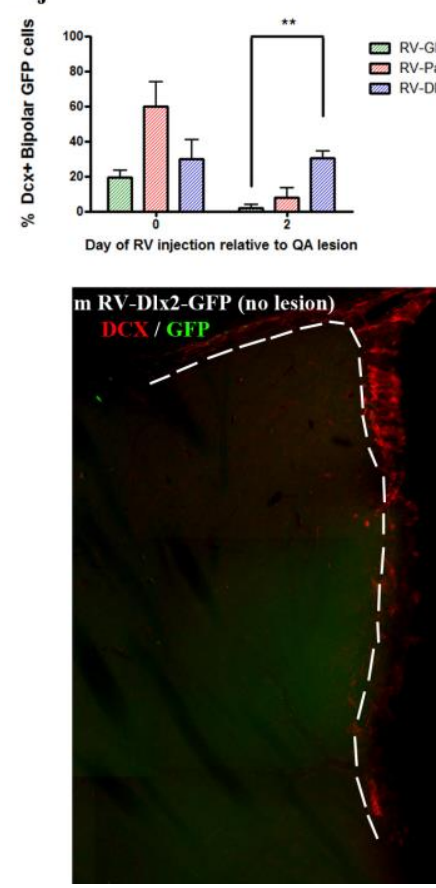

b

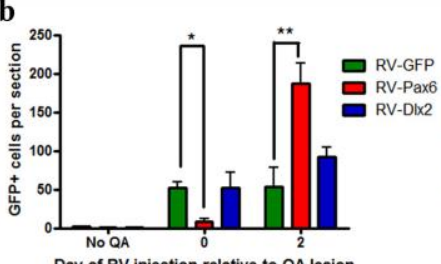

e

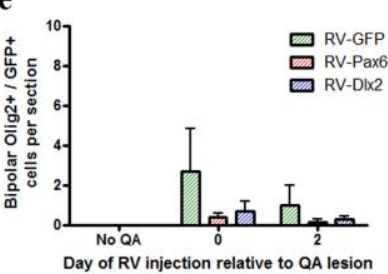

h

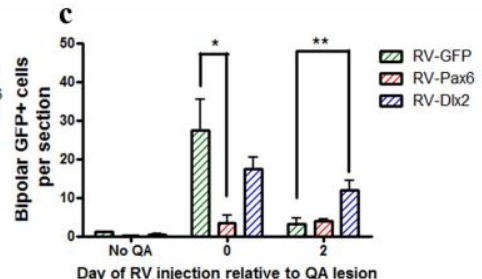

f

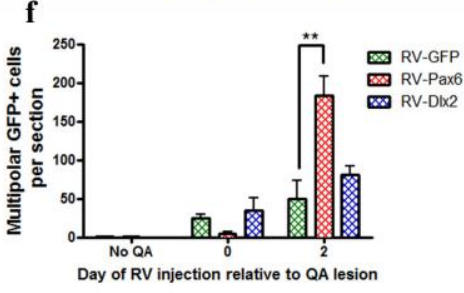

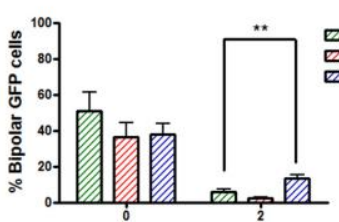

k

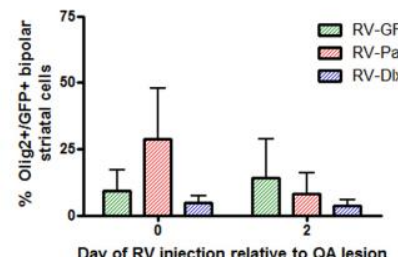

Day of RV injection relative to QA lesion

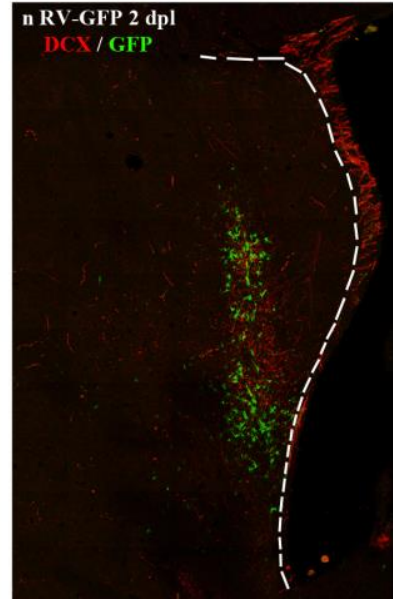

步

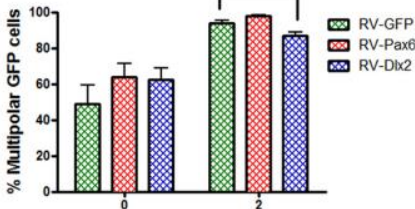

1 Day of RV injection relative to QA lesion
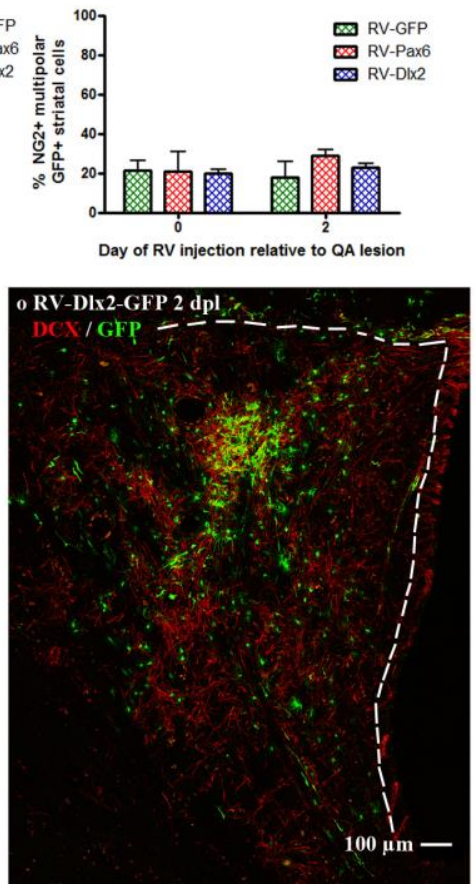

Figure 3: Ectopic expression of Pax6 or Dlx2 in SVZ-derived precursor cells following $Q A$ induced striatal lesioning alters GFP-labelled cell number, morphology and fate in the striatum. (a) Time line of $Q A$ and $R V$-Pax6 or RV-Dlx2 injections. (b-l) Graphs demonstrating: (b) the number of GFP-labelled cells per striatal section; (c) the number of bipolar GFP-labelled cells per striatal section; (d) the number of DCX/GFP cells per striatal section; (e) the number of bipolar OLIG2/GFP cells per striatal section; (f) the number of multipolar cells per striatal section: $(g)$ the number of NG2/GFP cells per striatal section; (h) the percentage of GFP-labelled cells exhibiting a bipolar morphology; (i) the percentage of GFP-labelled cells exhibiting a multipolar morphology; (j) the percentage of bipolar DCX/GFP cells; $(k)$ the percentage of bipolar OLIG2/GFP cells; and (l) the percentage of multipolar $N G 2 / G F P$ cells in the $Q A$ lesioned striatum (all one way ANOVAs * $P<0.05$; ** $P<0.01$. $(m-o)$ Images of GFP (green)/DCX (red) labelling from (m) RV-Dlx2-GFP in no-lesion controls (n) RV-GFP day 2 controls and (o) RV-Dlx2-GFP day 2 animals. Scale $m-o=100$ $\mu \mathrm{m}$. 
Interestingly, while both ectopic DLX2 and PAX6 could be detected in $83-92 \%$ of all RV-GFP labelled cells in SVZ of non-lesioned control animals (Supplementary Figure 4 a-c and 5 a-c), downregulation of transgenes was observed in the majority of GFP-labelled cells recruited into the striatum from animals injected at 2 days post lesion (Figure 4 a-f). Only 2-3\% of striatal GFP+ cells remained expressing PAX6 and DLX2. Similarly while Brgl, an essential gene for the pro-neurogenic function of bipolar SVZ PAX6 progenitor cells ${ }^{[38]}$, was observed in the SVZ of bipolar RV-PAX6-GFP cells
(Figure $4 \mathrm{~g}-\mathrm{j}$ ) and some GFP+ multipolar cells near to the RMS, it was absent in multipolar RV-PAX6-GFP+ cells in the striatum, of which some also stained for NG2 (Figure 4k, 1-s). This further indicates the loss of neuronal function in RV-PAX6 recruited cells. These findings indicate that $D l x 2$, while downregulated in many GFP cells of the striatum, does retain a significant effect on both the recruitment and regulation of DCX+ neuroblasts within the damaged striatum at acute time points post QA lesion.

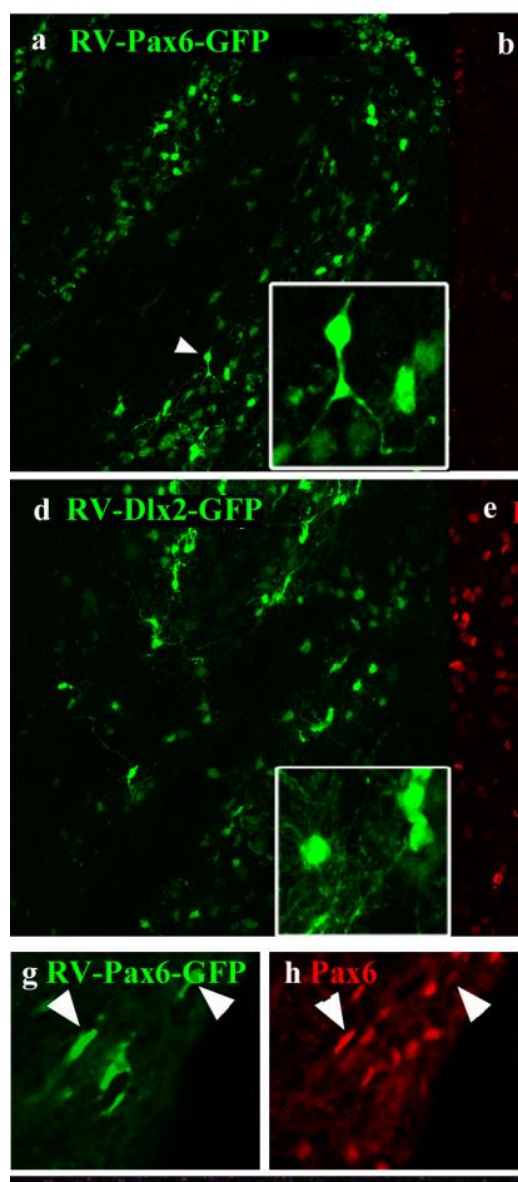

b Pax 6
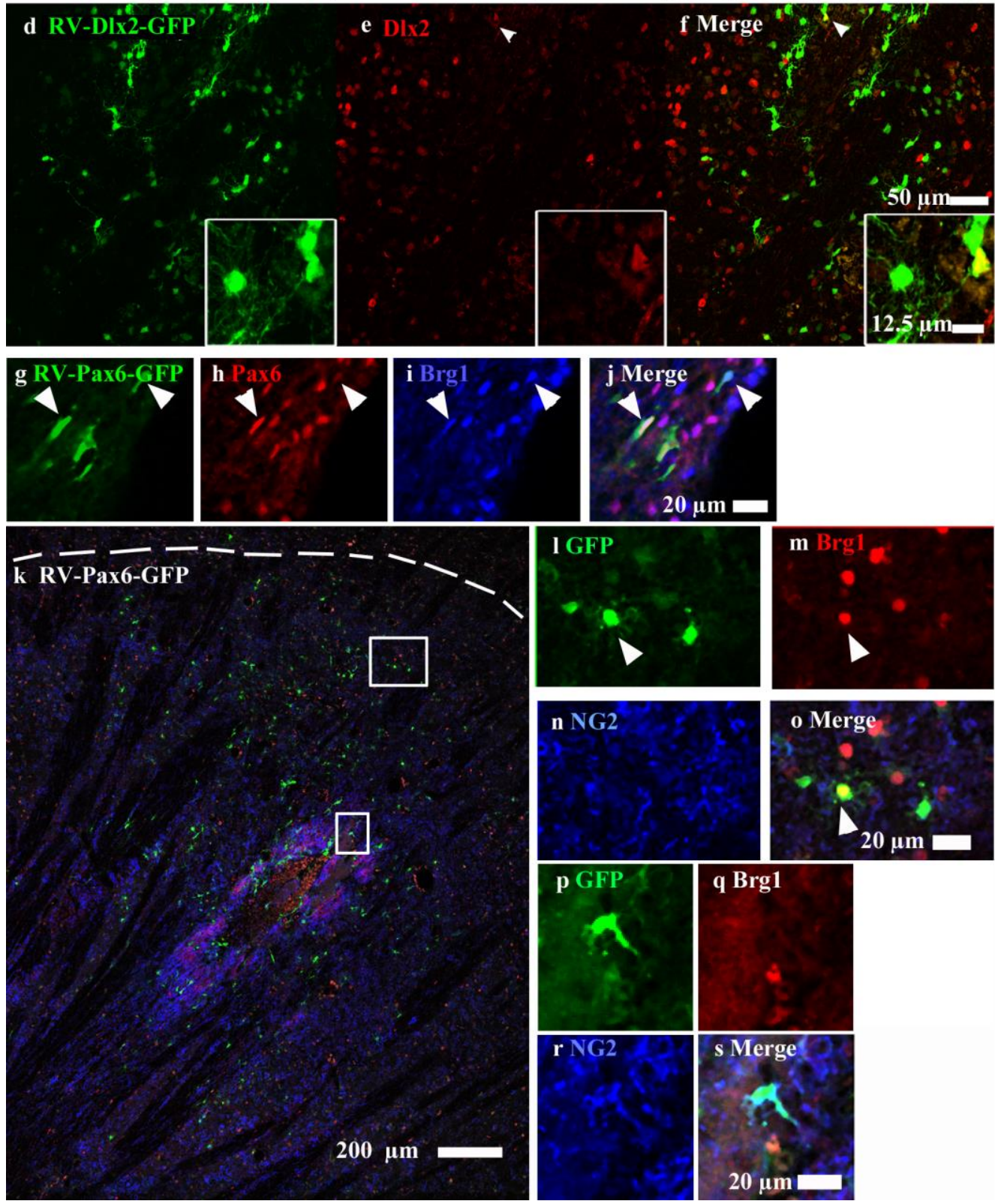

q Brg1
Figure 4: Down-regulation of transgene expression in the QA lesioned stratum and BRG1 expression.

PAX6 expression from RV-Pax6-GFP (a-c) and DLX2 expression from $R V$-Dlx2-GFP (df) is down-regulated in the lesioned striatum from cells labelled at $2 \mathrm{dpl}$. Arrowheads show few remaining positive co-expressing cells. ( $g$ j) Bipolar RV-Pax6-GFP cells (green) in the SVZ co-express PAX6 (red) and BRGI (blue). Arrowheads indicate two triple labelled cells. (k-s) In RV-Pax6-GFP animals, a few BRG+ multipolar GFP+ cells are observed close to the SVZ and RMS (l-o, upper boxed area), however all GFP+ multipolar cells further into the lesioned area and all NG2 / GFP+ cells are BRG negative ( $p$-s, lower boxed area). Scales a-f $=50 \mu \mathrm{m}$, boxed area $a-f 12.5$ $\mu \mathrm{m}, \mathrm{g}-\mathrm{j}$ and $\mathrm{l}-\mathrm{s}=20 \mu \mathrm{m}$ and $k=200 \mu \mathrm{m}$ 
The expression of signalling molecules in the SVZ following QAinduced striatal injury

Using quantitative PCR, we examined mRNA expression of a number of signalling molecules in the SVZ at 1,2,3 and 7 days post lesion in order to identify QA lesion-induced changes that may be implicated in the phenotypic switch of neural precursor cells in the lesioned environment. Changes in mRNA expression were observed in many of the main signalling families, including the BMP and Notch pathways and in molecules that are known to regulate the proliferation and migration of neural precursor cells (Table 1).
Bmp4, Heyl, Heyl, Tnr and pleiotropin mRNA expression was reduced in the first 1-2 days post QA lesion, then Bmp4, Tnr and pleiotropin significantly increased at 3 days post lesion, and Hes 1 at $7 \mathrm{dpl}$ when compared to unlesioned controls. Stat3 was increased throughout the time course, while Noggin was significantly increased at day 3. Further, a range of genes including Robol, Smad4 and $B M P 2$ were significantly reduced from day 1 up to days 3 to 7 post QA lesion. In addition, we observed a significant increase in the expression of $F g f 2$ and $B d n f$ mRNA from 1 to 3 days post QA lesion while expression of Fgfl3 and Vegf mRNA was reduced when compared to unlesioned controls.

\begin{tabular}{|c|c|c|c|c|c|c|c|c|}
\hline \multirow[b]{2}{*}{ Gene } & \multicolumn{2}{|c|}{$1 \mathrm{dpl}$} & \multicolumn{2}{|c|}{$2 \mathrm{dpl}$} & \multicolumn{2}{|c|}{3 dpl } & \multicolumn{2}{|c|}{$7 \mathrm{dpl}$} \\
\hline & Fold $\Delta$ & Sig. & Fold $\Delta$ & Sig. & Fold $\Delta$ & Sig. & Fold $\Delta$ & Sig. \\
\hline$B d n f$ & 11.4 & $*$ & 18.4 & * & 4.0 & * & 11.2 & ns \\
\hline$F g f 2$ & 4.1 & $* * *$ & 1.7 & * & 1.8 & $* *$ & 1.6 & ns \\
\hline Fgfl3 & -2.8 & ** & -1.7 & * & -3.2 & $* *$ & 1.3 & ns \\
\hline Vegf & -2.6 & $* * *$ & -1.9 & $* * *$ & -2.0 & $* * *$ & -1.7 & $* * *$ \\
\hline Bail & -3.3 & $* *$ & -1.4 & ** & -1.5 & $*$ & 1.1 & ns \\
\hline Artn & -1.8 & $* *$ & 1.0 & ns & -2.2 & $* *$ & 1.0 & ns \\
\hline Fez1 & -2.9 & $* * *$ & -1.6 & $* * *$ & -1.4 & $* *$ & -1.2 & ns \\
\hline Ptn & -1.6 & $* * *$ & 1.1 & ns & 1.7 & $* * *$ & 1.0 & ns \\
\hline$M d k$ & -1.3 & $* *$ & -1.5 & $* *$ & 1.1 & ns & 1.4 & ns \\
\hline Nrg1 & -2.1 & $* * *$ & 1.4 & ns & -2.9 & $* *$ & 1.1 & ns \\
\hline Втр2 & -3.0 & $* *$ & -3.6 & $* *$ & -2.2 & $*$ & -2.1 & * \\
\hline Bmp4 & -2.4 & $* *$ & -1.4 & ns & 2.4 & $* *$ & 1.7 & * \\
\hline Smad4 & -1.3 & $* *$ & -1.4 & $* * *$ & -1.2 & ns & 1.0 & ns \\
\hline Noggin & -1.6 & ns & 1.1 & ns & 1.8 & $* *$ & 1.3 & ns \\
\hline Stat3 & 4.2 & $* * *$ & 2.2 & $* *$ & 2.6 & $* * *$ & 2.0 & $* *$ \\
\hline Nrp2 & 2.3 & $* * *$ & 1.7 & $* *$ & 2.1 & $* *$ & 3.7 & ns \\
\hline Nrp1 & 1.1 & ns & 1.6 & ns & 2.0 & $* *$ & 2.1 & $*$ \\
\hline Robol & -2.7 & $* * *$ & -1.4 & $* *$ & -1.6 & $*$ & -1.1 & ns \\
\hline Ntn1 & -2.2 & $* * *$ & -1.5 & $* *$ & -1.2 & ns & -1.3 & ns \\
\hline Hes1 & 1.1 & $\mathrm{~ns}$ & 1.1 & ns & 1.5 & ns & 1.8 & $* *$ \\
\hline Heyl & -3.1 & $* * *$ & -1.7 & $* *$ & 1.1 & ns & -1.2 & ns \\
\hline Hey1 & -3.0 & $* * *$ & -1.8 & $* *$ & -1.1 & ns & -1.3 & ns \\
\hline Notch2 & 1.0 & ns & -1.1 & ns & 1.5 & ns & 1.3 & ns \\
\hline$T n r$ & -2.2 & $* * *$ & -2.1 & ns & 2.0 & $* * *$ & 1.2 & ns \\
\hline
\end{tabular}

Table 1: mRNA expression levels of signalling molecules in the SVZ following QA striatal lesioning. Fold changes shown in blue represent significant (sig.) downregulation greater than 1.5 fold compared to unlesioned control; fold changes shown in red represent significant up-regulation greater than 1.5 fold compared to unlesioned control. Sign. $=$ Significance $;$ dpl $=$ days post lesion; $* P<0.05, * * P<0.01, * * * P<0.001$, ns $=$ not significant. 


\section{Discussion}

While compensatory adult neurogenesis has been demonstrated in many models of brain injury, the correct cell types are not always generated for successful endogenous repair. We previously described transient SVZ precursor cell recruitment into the striatum following an excitotoxic injury, which exhibited a predominantly glial response $^{[6,25]}$. We have now confirmed that recruited cells of a bipolar morphology represent both neuroblasts and oligodendrocyte progenitor cells and that a proportion of multipolar cells are oligodendrocyte progenitors, but not astrocytes. Results observed in a Nestin-CreER ${ }^{\mathrm{T} 2}$ :R26R-YFP mouse stroke model reported similar percentages of recruited Dcx and NG2 progenitors as reported in our RV-GFP study, at 6 weeks post injury, but also identified a large percentage of GFAP+ astrocytes recruited into the striatum ${ }^{[39]}$. Astrocyte recruitment is not observed in our QA lesion model at the time points we examined, indicating endogenous repair mechanisms do vary between disease models and perhaps between species ${ }^{[39]}$.

Pro-neurogenic transcription factor delivery has been trialled as a mechanism to enhance the regenerative response to neural damage, with studies demonstrating moderate success when injecting proneurogenic transcription factors directly into damaged brain parenchyma $^{[12,17-19,27,28]}$. The current study therefore aimed to convert the observed gliogenic response to a neurogenic one by individually over-expressing the pro-neurogenic genes Pax6 or Dlx2 in SVZ-derived neural precursors. Interestingly we found that only ectopic Dlx2 expression was able to overcome signals released from damaged tissue and enhance both neuroblast recruitment and the percentage of recruited cells that retained a neuronal fate in the QA lesioned striatum. We propose this may reflect the importance the microenvironment plays in regulating neural cell fate following injury or disease.

While it is believed that Dlx2 and Pax6 may have similar roles in promoting adult $\mathrm{SVZ}$ neurogenesis ${ }^{[12,40]}$, very different results were observed in the current study following Dlx2 over-expression when compared to Pax6. This may be explained by different complements of chemokine receptors on PAX6+ and DLX2+ precursor cells, as inflammatory-induced chemoattractants play an essential role in neuroblast and OPC recruitment ${ }^{[3,41-44]}$ and have been shown to be expressed in the QA lesioned striatum ${ }^{[41,42]}$. While DLX2+ precursor cells have been observed to migrate towards chemoattractants during forebrain development ${ }^{[45]}$, expression of chemokine receptors specifically on adult SVZ-derived PAX6+ or DLX2+ precursor cells has never has been examined. We thus propose the pan-cell type decrease in Pax6-GFP cells observed in the striatum of animals labelled at the time of QA lesioning may indicate that RV-Pax6 overexpression has generated PAX6+ precursor cells (possibly TAPs) which either do not express the complement of chemokine receptors required to respond to migratory cues released from the QA lesioned striatum at the time of lesioning or chemokines that attract PAX6+ precursor cells may not be secreted from the QA lesioned striatum at this immediate time point. Lack of appropriate chemokine signalling is likely because PAX6+ cells labelled two days post lesioning were recruited in large numbers to lesioned striatum. It is also possible that the RV-Pax6 cells are recruited into the striatum to a similar extent as the GFP and RV-Dlx2 cells, but do not survive at the early time point, while increasing proliferation at the latter time point. Further analysis is required however to identify the chemokine/signalling molecule(s) responsible for this Pax6-dependant effect.
In contrast, Dlx 2 over-expression at both time points examined did not lead to a change in the number of recruited GFP+ cells to the striatum, suggesting these cells may be responding to a different chemokine or complement of chemokines. Over-expression of Dlx2 has been shown to promote reprogramming of adult cortical astroglia into functional GABAergic neurons in vitro, under both normal conditions and in cortical astrocytes responding to injury ${ }^{[46,47]}$. Dlx2 is also believed to repress oligodendrogenesis by suppressing Olig2 in the adult SVZ through a BMP mediated mechanism ${ }^{[31]}$. Consistent with this, RV-DLX2 expression did increase total bipolar GFP+ cell recruitment and DCX+ cell number, in conjunction with a reduction in multipolar GFP+ cell number when compared to RV-GFP control animals. However, as OLIG2+ and NG2+ cell numbers remained unchanged this suggests that instead of suppressing Olig2 expression, Dlx2 may be altering the fate of the lineage negative GFP+ cells identified in controls. While DCX has been observed to co-label with $\mathrm{NG} 2$ in the cortex ${ }^{[48]}$, confocal imaging confirmed that DCX+ neuroblasts in the SVZ and migrating into the lesioned striatum do not express NG2, establishing their neuronal fate in the striatum. Unfortunately, staining for mature neuronal markers was not able to be performed due to the immature fate of the cells. Therefore, in contrast to Pax6, Dlx2 over-expression in SVZ precursor cells has a significant neurogenic effect within the QA lesioned striatum.

In contrast to previous work ${ }^{[18,19]}$, we observed that over-expression of Pax6 in SVZ-derived precursor cells was unable to promote an increase in DCX+ cell recruitment in the QA lesioned striatum. Instead, ectopic PAX6 expression appeared to promote an oligo-glial cell fate. Ninkovic and colleagues ${ }^{[38]}$ have reported that adult SVZ neurogenesis regulated by Pax6 is co-dependant on a Brg-containing BAF chromatin remodelling complex. Loss of either Pax6 or Brgl alters the fate of adult SVZ-derived precursor cells. Specifically, in the parenchyma surrounding the SVZ and RMS, loss of Pax6 or Brgl altered neuroblast lineage to OLIG2+ and/or NG2+ OPCs, starting in the RMS and this effect was specific to PAX6 progenitors. While we saw positive staining of RV-PAX6-GFP cells with PAX6 and BRG1 in the SVZ, we also observed an absence of PAX6 and BRG1 in RVPAX6-GFP cells in the striatum that acquired a multipolar fate and some of which were positive for NG2. This suggests signals released after QA lesioning may be promoting the alteration of SVZ-derived Pax6-expressing cells to an oligodendroglial fate, resulting in the reduction in DCX+ neuroblasts observed. We also observed some BRG1+ immunostaining for RV-DLX2-GFP cells in both bipolar and multipolar cells of the striatum (data not shown), indicating a different regulatory role for Brgl to the one it plays for Pax6. This is unsurprising as Brgl control of neurogenesis was found to be specific for $\operatorname{Pax}^{[38]}$. Jablonska and colleagues (2010) also found alterations in BMP signals in the SVZ which led PAX6+ neuronal precursor cells to switch their molecular, cellular and migratory paths towards an oligodendrocyte lineage following white matter injury $^{[24]}$. In the current study we also observed alterations in SVZ BMP signalling following QA lesion that could have impacted the increase in glial cell fate found with RV-Pax6. In addition to dynamic changes in Bmp2 and Bmp4 expression, we found significant up-regulation of Stat3, Fgf2, Bdnf, Hesl, and Noggin. These changes were coupled with significant decreases in many other molecules and growth factors indicating the complex environmental regulation within the $S V Z$ precursor pool after injury.

It is interesting to observe such dynamic changes in signalling molecules in the SVZ after a striatal lesion, but it is difficult to fully understand how or in fact which of these molecules are acting 
specifically on the Dlx2 and Pax6 TAPS or neuroblasts of the SVZ, as to date very few studies have been performed to examine the range of receptors on these cells. Further, when tissue is collected for gene expression analysis the entire SVZ is pooled, resulting in a heterogeneous mix of all cell types. This means only the 'big picture' and not effects on specific cell types can be observed. Further studies are needed to tease out the specific effects of each signalling factor on the individual cells of the SVZ.

Interestingly, two recent papers have identified parenchymal astrocytes in the mouse striatum that can respond to QA lesion or stroke induced signals, up-regulate Mashl and generate DCX+ neuroblasts contributing to striatal regeneration ${ }^{[8,9]}$. A role for decreased Notch signalling within these cells in the striatum was implicated for this endogenous repair mechanism. We also observed down-regulation in some components of the Notch signalling pathway in the SVZ immediately following lesioning, further supporting that lesion-induced cues can alter Notch regulation of SVZ neurogenesis. It is exciting to note that the rodent brain appears to retain the capacity for both acute recruitment of cells from the SVZ immediately following injury (within the first 2 days following QA lesion) and a longer term ability to regenerate neuroblasts from striatal astrocytes activating a neurogenic programme (from 2 weeks post injury $\left.)^{[8,9}, 25,39\right]$. Our study only examined acute time points following striatal lesioning which may be why we did not observe parenchymal astrocyte neurogenesis. Long term survival and differentiation studies on RV-Pax6 and RV-Dlx2 cells will offer more information on fate-switching and the endogenous repair potential from both recruited SVZ cells and neurogenic striatal astrocytes.

\section{Conclusion}

The current study demonstrates that RV-Dlx2 over-expression in SVZ-derived progenitors can stimulate acute neuroblast recruitment and retention of a neuronal fate in an excitotoxic lesion model at a previously non-neurogenic time point. In contrast, over-expression of Pax6 in SVZ-derived progenitor cells was not successful at inhibiting an oligodendrocyte fate and resulted in increased multipolar cell numbers with no change in neuronal recruitment. This indicates a complex interaction between signals released from the lesioned environment and gene expression in recruited cells. To promote successful endogenous repair after injury, we first need to better understand which lesion-induced signals are recruiting the subsets of SVZ progenitors into the striatum, and also how they are altering cell fate determination during this endogenous repair process.

\section{References}

1. Curtis MA, Penney EB, Pearson AG, van Roon-Mom WM, Butterworth NJ, Dragunow M, Connor B, Faull RL. Increased cell proliferation and neurogenesis in the adult human Huntington's disease brain. Proc Natl Acad Sci U S A. 2003; 100(15): 9023-27.

2. Jones KS, Connor B. Proneural transcription factors Dlx 2 and Pax6 are altered in adult SVZ neural precursor cells following striatal cell loss. Mol Cell Neurosci. 2011; 47(1): 53-60.

3. Cayre M, Canoll P, Goldman JE. Cell migration in the normal and pathological postnatal mammalian brain. Prog Neurobiol. 2009; 88(1): 41-63.

4. Kernie SG, Parent JM. Forebrain neurogenesis after focal Ischemic and traumatic brain injury. Neurobiol Dis. 2010; 37(2): 267-74.

5. Zhang RL, Zhang ZG, Chopp M. Ischemic stroke and neurogenesis in the subventricular zone. Neuropharmacology. 2008; 55(3): 34552.

6. Tattersfield AS, Croon RJ, Liu YW, Kells AP, Faull RL, Connor B. Neurogenesis in the striatum of the quinolinic acid lesion model of Huntington's disease. Neuroscience. 2004; 127(2): 319-32.
7. Ninkovic J, Gotz M. Fate specification in the adult brain--lessons for eliciting neurogenesis from glial cells. Bioessays. 2013; 35(3): 242-52.

8. Nato G, Caramello A, Trova S, Avataneo V, Rolando C, Taylor V, Buffo A, Peretto P, Luzzati F. Striatal astrocytes produce neuroblasts in an excitotoxic model of Huntington's disease. Development. 2015; 142(5): 840-45.

9. Magnusson JP, Goritz C, Tatarishvili J, Dias DO, Smith EM, Lindvall O, Kokaia Z, Frisen J. A latent neurogenic program in astrocytes regulated by Notch signaling in the mouse. Science. 2014; 346(6206): 237-41.

10. Alvarez-Buylla A, Garcia-Verdugo JM. Neurogenesis in adult subventricular zone. J Neurosci. 2002; 22(3): 629-34.

11. Parras CM, Galli R, Britz O, Soares S, Galichet C, Battiste J, Johnson JE, Nakafuku M, Vescovi A, Guillemot F. Mash1 specifies neurons and oligodendrocytes in the postnatal brain. Embo J. 2004; 23(22): 4495-4505.

12. Brill MS, Snapyan M, Wohlfrom H, Ninkovic J, Jawerka M, Mastick GS, Ashery-Padan R, Saghatelyan A, Berninger B, Gotz M. A dlx2- and pax6-dependent transcriptional code for periglomerular neuron specification in the adult olfactory bulb. $\mathbf{J}$ Neurosci. 2008; 28(25): 6439-52.

13. Hack MA, Saghatelyan A, de Chevigny A, Pfeifer A, AsheryPadan R, Lledo PM, Gotz M. Neuronal fate determinants of adult olfactory bulb neurogenesis. Nat Neurosci. 2005; 8(7): 865-72.

14. Roybon L, Deierborg T, Brundin P, Li JY. Involvement of Ngn2, Tbr and NeuroD proteins during postnatal olfactory bulb neurogenesis. Eur J Neurosci. 2009; 29(2): 232-43.

15. Kohwi M, Osumi N, Rubenstein JL, Alvarez-Buylla A. Pax6 is required for making specific subpopulations of granule and periglomerular neurons in the olfactory bulb. J Neurosci. 2005; 25(30): 6997-7003.

16. Brill MS, Ninkovic J, Winpenny E, Hodge RD, Ozen I, Yang R, Lepier A, Gascon S, Erdelyi F, Szabo G, Parras C, Guillemot F, Frotscher M, Berninger B, Hevner RF, Raineteau O, Gotz M. Adult generation of glutamatergic olfactory bulb interneurons. Nat Neurosci. 2009; 12(12): 1524-33.

17. Zhang RL, Chopp M, Roberts C, Jia L, Wei M, Lu M, Wang X, Pourabdollah S, Zhang ZG. Ascl1 lineage cells contribute to ischemia-induced neurogenesis and oligodendrogenesis. J Cereb Blood Flow Metab. 2011; 31(2): 614-25.

18. Kronenberg G, Gertz K, Cheung G, Buffo A, Kettenmann H, Gotz M, Endres M. Modulation of fate determinants Olig2 and Pax6 in resident glia evokes spiking neuroblasts in a model of mild brain ischemia. Stroke. 2010; 41(12): 2944-49.

19. Buffo A, Vosko MR, Erturk D, Hamann GF, Jucker M, Rowitch D, Gotz M. Expression pattern of the transcription factor Olig2 in response to brain injuries: implications for neuronal repair. Proc Natl Acad Sci U S A. 2005; 102(50): 18183-188.

20. Wei B, Nie Y, Li X, Wang C, Ma T, Huang Z, Tian M, Sun C, Cai Y, You Y, Liu F, Yang Z. Emx1-expressing neural stem cells in the subventricular zone give rise to new interneurons in the ischemic injured striatum. Eur J Neurosci. 2011; 33(5): 819-30.

21. Yang Z, You Y, Levison SW. Neonatal hypoxic/ischemic brain injury induces production of calretinin-expressing interneurons in the striatum. J Comp Neurol. 2008; 511(1): 19-33.

22. Arvidsson A, Collin T, Kirik D, Kokaia Z, Lindvall O. Neuronal replacement from endogenous precursors in the adult brain after stroke. Nat Med. 2002; 8(9): 963-70.

23. Parent JM, Vexler ZS, Gong C, Derugin N, Ferriero DM. Rat forebrain neurogenesis and striatal neuron replacement after focal stroke. Ann Neurol. 2002; 52(6): 802-13.

24. Jablonska B, Aguirre A, Raymond M, Szabo G, Kitabatake Y, Sailor KA, Ming GL, Song H, Gallo V. Chordin-induced lineage plasticity of adult SVZ neuroblasts after demyelination. Nat Neurosci. 2010; 13(5): 541-50.

25. Gordon RJ, Tattersfield AS, Vazey EM, Kells AP, McGregor AL, Hughes SM, Connor B. Temporal profile of subventricular zone progenitor cell migration following quinolinic acid-induced striatal cell loss. Neuroscience. 2007; 146(4): 1704-18.

26. Thored P, Arvidsson A, Cacci E, Ahlenius H, Kallur T, Darsalia V, Ekdahl CT, Kokaia Z, Lindvall O. Persistent production of neurons from adult brain stem cells during recovery after stroke. Stem Cells. 2006; 24(3): 739-47. 
27. Tepavcevic V, Lazarini F, Alfaro-Cervello C, Kerninon C, Yoshikawa K, Garcia-Verdugo JM, Lledo PM, NaitOumesmar B, Baron-Van Evercooren A. Inflammationinduced subventricular zone dysfunction leads to olfactory deficits in a targeted mouse model of multiple sclerosis. J Clin Invest. 2011; 121(12): 4722-34.

28. Grande A, Sumiyoshi K, Lopez-Juarez A, Howard J, Sakthivel B, Aronow B, Campbell K, Nakafuku M. Environmental impact on direct neuronal reprogramming in vivo in the adult brain. Nat Commun. 2013; 4: 2373.

29. Hack MA, Sugimori M, Lundberg C, Nakafuku M, Gotz $M$. Regionalization and fate specification in neurospheres: the role of Olig2 and Pax6. Mol Cell Neurosci. 2004; 25(4): 664-78.

30. Heins N, Malatesta P, Cecconi F, Nakafuku M, Tucker KL, Hack MA, Chapouton P, Barde YA, Gotz M. Glial cells generate neurons: the role of the transcription factor Pax6. Nat Neurosci. 2002; 5(4): 308-15.

31. Colak D, Mori T, Brill MS, Pfeifer A, Falk S, Deng C, Monteiro R, Mummery C, Sommer L, Gotz M. Adult neurogenesis requires Smad4-mediated bone morphogenic protein signaling in stem cells. J Neurosci. 2008; 28(2): 434-46.

32. Livak KJ, Schmittgen TD. Analysis of relative gene expression data using real-time quantitative PCR and the 2(-Delta Delta C(T)) Method. Methods. 2001; 25(4): 40208 .

33. Miller DG, Adam MA, Miller AD. Gene transfer by retrovirus vectors occurs only in cells that are actively replicating at the time of infection. Mol Cell Biol. 1990; 10(8): 4239-42.

34. Aguirre A, Gallo V. Reduced EGFR signaling in progenitor cells of the adult subventricular zone attenuates oligodendrogenesis after demyelination. Neuron Glia Biol. 2007; 3(3): 209-20.

35. Ming GL, Song H. Adult neurogenesis in the mammalian central nervous system. Annu Rev Neurosci. 2005; 28 : 223-50.

36. Lewis PF, Emerman M. Passage through mitosis is required for oncoretroviruses but not for the human immunodeficiency virus J Virol. 1994;68(1):510-6.

37. Nishiyama A. Polydendrocytes: NG2 cells with many roles in development and repair of the CNS. Neuroscientist. 2007; 13(1): 62-76.

38. Ninkovic J, Steiner-Mezzadri A, Jawerka M, Akinci U, Masserdotti G, Petricca S, Fischer J, von Holst A, Beckers J, Lie CD, Petrik D, Miller E, Tang J, Wu J, Lefebvre V, Demmers J, Eisch A, Metzger D, Crabtree G, Irmler M, Poot R, Gotz M. The BAF Complex Interacts with Pax6 in Adult Neural Progenitors to Establish a Neurogenic Cross-Regulatory Transcriptional Network. Cell Stem Cell. 2013; 13(4): 403-18.
39. Li L, Harms KM, Ventura PB, Lagace DC, Eisch AJ, Cunningham LA. Focal cerebral ischemia induces a multilineage cytogenic response from adult subventricular zone that is predominantly gliogenic. Glia. 2010; 58(13): 1610-19.

40. de Chevigny A, Core N, Follert P, Wild S, Bosio A, Yoshikawa K, Cremer H, Beclin C. Dynamic expression of the pro-dopaminergic transcription factors Pax6 and Dlx2 during postnatal olfactory bulb neurogenesis. Front Cell Neurosci. 2012; 6: 6.

41. Connor B, Gordon RJ, Jones KS, Maucksch C. Deviating from the well travelled path: Precursor cell migration in the pathological adult mammalian brain. J Cell Biochem. 2011; 112(6): 1467-74.

42. Gordon RJ, McGregor AL, Connor B. Chemokines direct neural progenitor cell migration following striatal cell loss. Mol Cell Neurosci. 2009; 41(2): 219-32.

43. Tsai HH, Frost E, To V, Robinson S, Ffrench-Constant C, Geertman R, Ransohoff RM, Miller RH. The chemokine receptor CXCR2 controls positioning of oligodendrocyte precursors in developing spinal cord by arresting their migration. Cell. 2002; 110(3): 373-83

44. Banisadr G, Frederick TJ, Freitag C, Ren D, Jung H, Miller SD, Miller RJ. The role of CXCR4 signaling in the migration of transplanted oligodendrocyte progenitors into the cerebral white matter. Neurobiol Dis. 2011; 44(1): 19-27.

45. Tiveron MC, Rossel M, Moepps B, Zhang YL, Seidenfaden R, Favor J, Konig N, Cremer H. Molecular interaction between projection neuron precursors and invading interneurons via stromal-derived factor 1 (CXCL12)/CXCR4 signaling in the cortical subventricular zone/intermediate zone. J Neurosci. 2006; 26(51): 13273-78.

46. Blum R, Heinrich C, Sanchez R, Lepier A, Gundelfinger $\mathrm{ED}$, Berninger B, Gotz M. Neuronal network formation from reprogrammed early postnatal rat cortical glial cells. Cereb Cortex. 2010; 21(2): 413-24.

47. Heinrich C, Blum R, Gascon S, Masserdotti G, Tripathi P, Sanchez R, Tiedt S, Schroeder T, Gotz M, Berninger B. Directing astroglia from the cerebral cortex into subtype specific functional neurons. PLoS Biol. 2010; 8(5): e10000373.

48. Tamura Y, Kataoka Y, Cui Y, Takamori Y, Watanabe Y, Yamada H. Multi-directional differentiation of doublecortin- and NG2-immunopositive progenitor cells in the adult rat neocortex in vivo. Eur J Neurosci. 2007; 25(12): 3489-98.
Abbreviations
RV: $\quad$ Retroviral
SVZ: Subventricular zone
OPC : Oligodendrocyte precursor cells
TAPs : Transiently Amplifying Precursors
NPCs : Neural Progenitor Cells
RV : $\quad$ Retrovirus
QA : Quinolinic Acid
DPL: Days post lesion
DPI: Days post injection 


\section{Potential Conflicts of Interests}

\section{None}

\section{Acknowledgments}

The authors would like to thank Professor Magdalena Gotz for kindly donating the plasmids for use in this study.

\section{Sponsors / Grants}

Funding was provided by the Neurological Foundation of New Zealand (66 Grafton Rd, Grafton, Auckland, New Zealand), and the Health Research Council of New Zealand (Level 3, 110 Stanley St, Grafton, Auckland 1010). Jones KS was supported by a Neurological Foundation of NZ Postgraduate Scholarship.

\section{Additional Information}

Supplementary Information accompanies this article. Supplementary figures are linked to the online version of the article.

\section{Corresponding Author}

Bronwen J Connor, Centre for Brain Research, Department of Pharmacology and Clinical Pharmacology, School of Medical Science, Faculty of Medical and Health Sciences, University of Auckland, 85 Park Road, Grafton, Auckland, New Zealand 1023. Email: $\underline{\text { b.connor@auckland.ac.nz }}$ 